\title{
Regulations and ethics surrounding cluster randomized controlled trials and other clinical trials in emergency medicine
}

Toshihiro Hatakeyama ${ }^{1 \#}$, Tashiro Shimon ${ }^{2}$

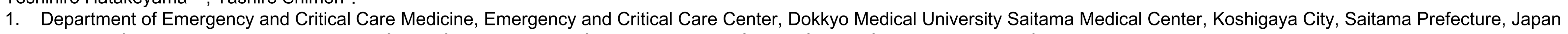

2. Division of Bioethics and Healthcare Law, Center for Public Health Sciences, National Cancer Center, Chuo-ku, Tokyo Prefecture, Japan

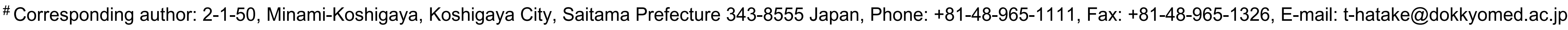

Introduction

A cluster randomized controlled trial (CRT) is performed as an intervention in a group of subjects (collectively labeled as a 'cluster'), rather than for an individual. ${ }^{1}$ Because of an unique feature of this study design, it can be difficult to apply standard research ethics guidelines to CRT. Several authors have documented challenges related to obtaining informed consent (IC) of CRT. The 2012 Ottawa Statement on the Ethical Design and Conduct of Cluster Randomized Trials identifies important issues related to CRTs in general and offers recommendations at the intersection of ethics and CRTs. ${ }^{2}$

However, Japan has yet to create an established guideline for CRT including how to obtain IC. Recently, we have made the study protocol for CRT on resuscitation science in Japan. ${ }^{3}$ The aim of this study was to share this experience and suggest a method for obtaining IC in CRT of resuscitation science in Japan and other regions.

Case

Cardiopulmonary resuscitation (CPR) feedback device to be used in our study is mounted on the defibrillator and is equipped with an accelerometer, to monitor the motion of the patient's sternum and indicate CPR quality on the scene (real-time feedback). This CPR feedback device also allows emergency medical services (EMS) personnel to review recorded CPR quality that is evaluated based on the quantified fraction, depth, and tempo for use in debriefing and physical retraining after the event. The CPR feedback devices are randomly assigned to the intervention group or the control group in each EMS station (Figure 1)

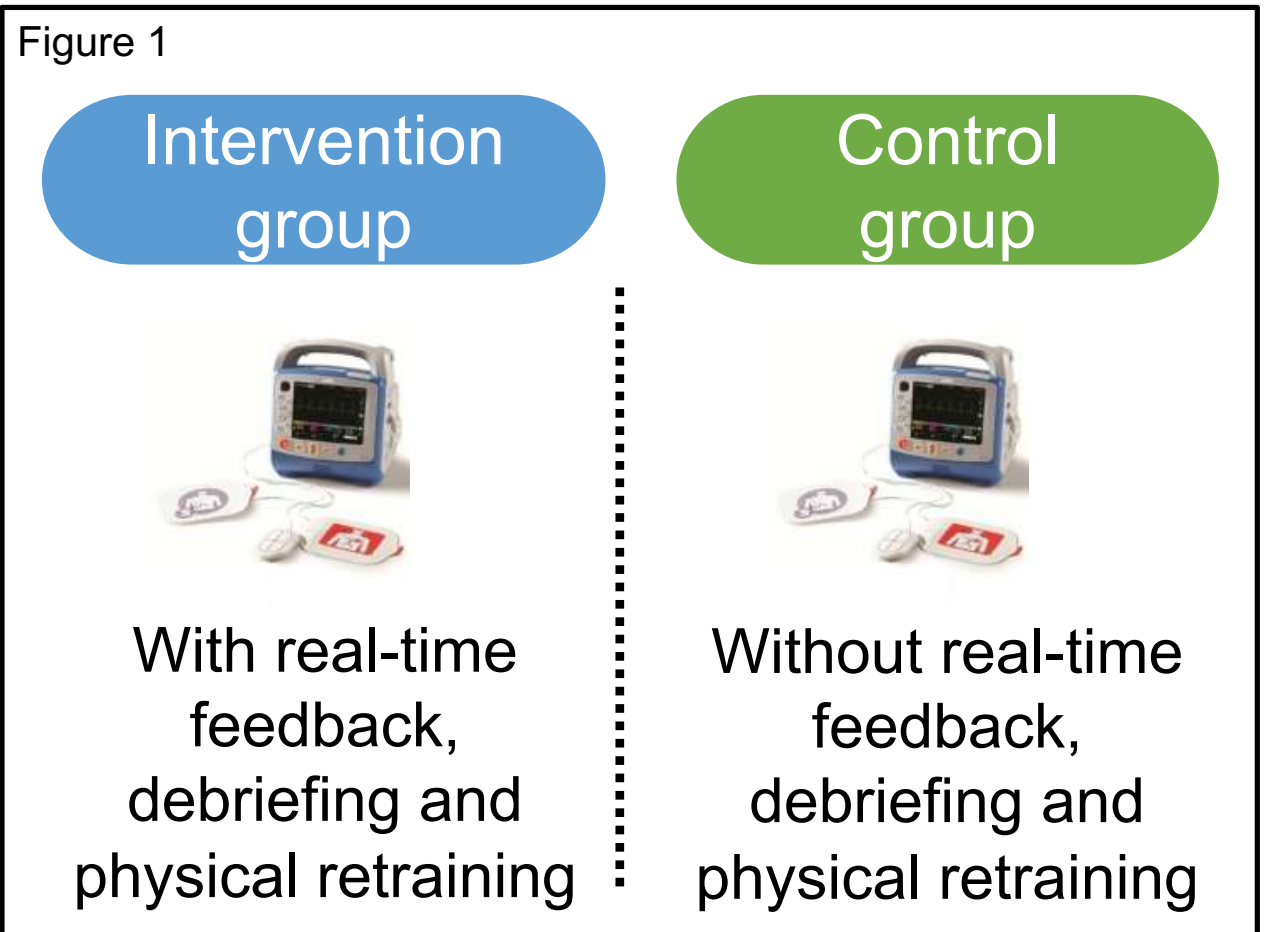

This study is designed as a CRT and conducted at three fire departments with 32 EMS stations, covering five municipalities in Osaka Prefecture, Japan (Figure 2).

We will examine whether the combination of real-time feedback, debriefing, and retraining using a CPR feedback device will outperform conventional treatment without any feedback, debriefing, or retraining in terms of favorable neurological survival among cardiac arrest patients who receive CPR by EMS personnel.

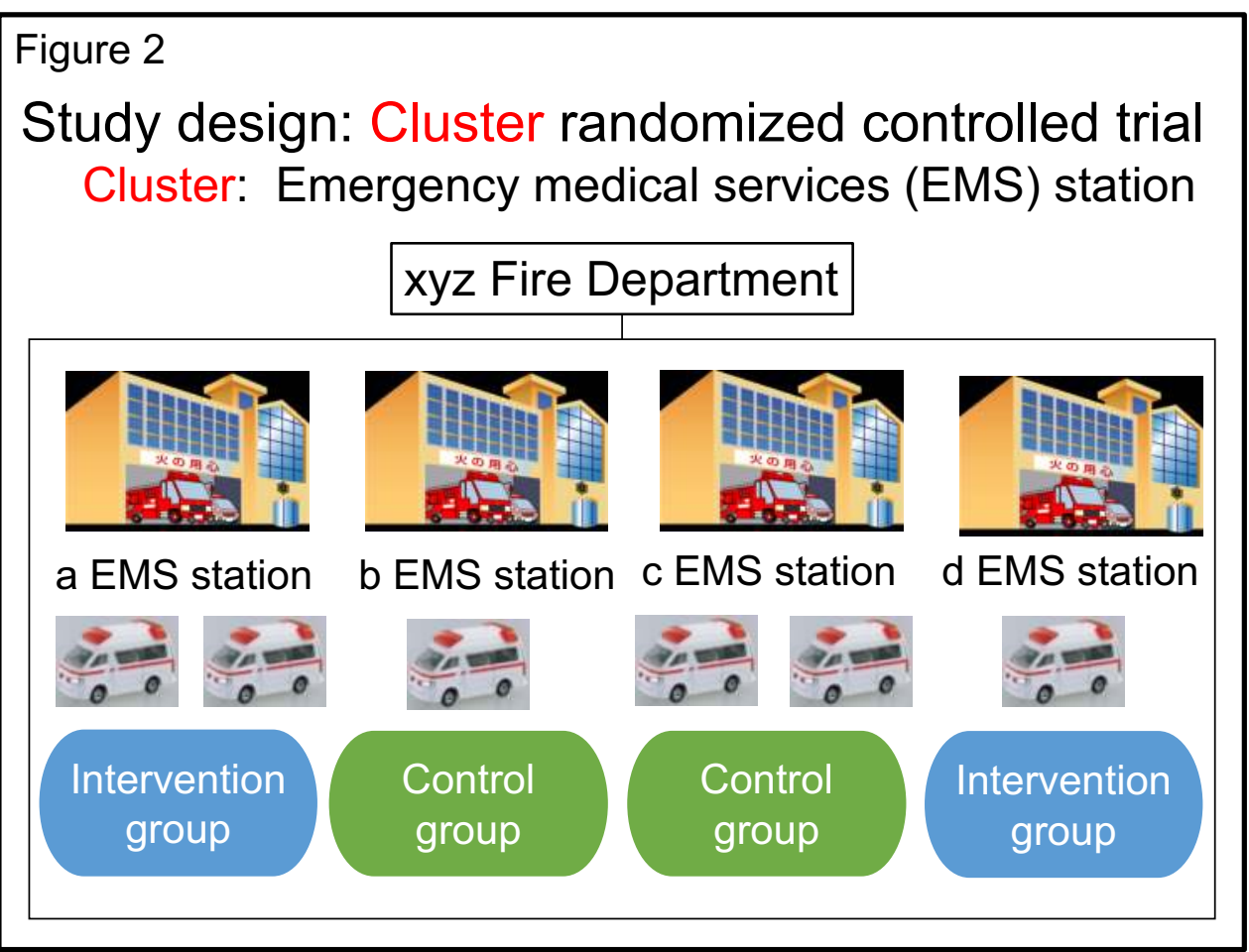

This trial is registered in the University Hospital Medical Information Network (UMIN) Clinical Trials Registry with number UMIN000021431 on the $11^{\text {th }}$ March, 2016.

\section{Ethical issues in CRT}

Before the protocol of this trial has been approved by the ethics committee of Kyoto University Graduate School of Medicine (registration number C-1154), we discussed the following ethical issues in this study and other CRTs including emergency medicine.

determine who are the research participants and what other individuals or groups are affected, even though they are not directly targeted;

determine whether it is required or feasible to obtain IC from patients, health care workers, or community members; and

- determine whether requiring informed consent and allowing refusal to consent may invalidate or compromise the research results.

\section{Suggestions for further CRTs}

We reviewed previous literature on research ethics and international regulations to identify the core features of IC in CRT of resuscitation science. Our suggestions on ethical issues for further CRTs in Japan and other regions are shown as Figure 3 .

\section{Figure 3}

A waiver or modification of IC on CRTs may be approved when the following requirements are filled. - The research is not feasible without a waiver or alteration of consent

- The study interventions and data collection procedures pose no more than minimal risk. - A waiver or modification of IC is not against research participants' potential interests.

Institutional review boards should evaluate whether study design is optimal.

- Researchers must obtain IC from gatekeepers, such as a community leader, headmaster, or loca health council unless conditions for a waiver or alteration of consent are met.

\section{Discussions}

- We believe that waivers or modifications of IC may be necessary in some clinical research on CRTs in which it is essentially impossible to obtain individual IC.

- If needed, a gatekeeper should be chosen to represent a wider group of community representatives or advisers before taking the decision to permit the study (Figure4).

Figure 4

\section{-Gatekeeper}

Gatekeepers are individuals or bodies who may be called upon to protect the group-based interests that are affected by enrollment in a CRT.

$>$ When CRT interventions may substantially affect cluster interests, researchers should seek to protect cluster interests through cluster consultation to inform study design, conduct, and reporting. Where relevant, gatekeepers can often facilitate such a consultation.

\section{Conclusions}

To support studies of CRTs including resuscitation science, we shared our experience through the large CRT focusing pre-hospital CPR in Japan and presented suggestions on ethical issues.

\section{Main references}

1. International Ethical Guidelines for Healthrelated Research Involving Humans. (https://cioms.ch/wp-

content/uploads/2017/01/WEB-CIOMSEthicalGuidelines.pdf)

2. The Ottawa Statement on the Ethical Design and Conduct of Cluster Randomized Trials. PLoS Med. 9(11): e1001346.

3. Real-time feedback, debriefing, and retraining system of cardiopulmonary resuscitation for out-of-hospital cardiac arrest: a study protocol; for a cluster parallel-group randomized controlled trial. Trials. in press. 